\title{
Discovery of genetic polymorphism: Richard Lewontin and Tomoko Ohta awarded the Crafoord Prize in Biosciences 2015
}

\author{
Bo Söderström
}

Published online: 25 February 2015

Richard Lewontin and Tomoko Ohta are awarded the 2015 Crafoord Prize for their pioneering analyses and fundamental contributions to the understanding of genetic polymorphism. Earlier Crafoord laureates in biosciences include Ilkka Hanski (2011), Robert L. Trivers (2007), Carl R. Woese (2003), John Maynard Smith, Ernst Mayr and George C. Williams (1999), Robert May (1996), Seymour Benzer and William T. Hamilton (1993), Paul R. Ehrlich and Edward O. Wilson (1990), Eugene P. Odum and Howard T. Odum (1987), and Daniel H. Janzen (1984). The scientific discoveries of Richard Lewontin and Tomoko Ohta are of great general significance in basic research but applied knowledge of genetic variation is highly relevant also in environmental and conservation sciences.

In ecology and conservation, it has led to a better understanding of population structure and genetic vulnerability among threatened populations. Another result has been new methods for estimating the size of natural populations using the DNA analysis of animal spoor, such as scat. In systematic biology, knowledge of genetic variation has resulted in new opportunities for understanding relationships between and within species. Furthermore, it is now possible to determine close relationships between individuals using DNA analysis. In many species, the females mate with more than one male, so that the offspring in one litter are often half-siblings.

Nowadays, it may seem obvious that our genetic makeup is as unique as our fingerprints, but this was a contradiction to the prevailing theories when it was discovered in the 1960s. The view of genetic variation up until then was entirely different: biologists believed that most individuals in a population were fairly similar, genetically speaking. This must, they assumed, be the result of natural selection, where every genetic variant that was less beneficial was eliminated. Richard Lewontin used a method that separated proteins based on their molecular characteristics, obtaining very surprising results. The genetic variation between individuals in a population was many times greater than expected. The pattern was repeated in every species examined. They all demonstrated a significant and unexpected genetic variation, appearing to contradict the principles of natural selection. A theory of neutral mutations was put forward, in which gene variants that neither improve nor worsen an individual's fitness are created.

Geneticist Tomoko Ohta developed theory suggesting that almost all mutations in genes that affect the encoded proteins are somewhat harmful, but the effect of this is so small that these gene variants can remain in the population. They can thus be considered nearly neutral. Ohta also showed that the size of a population is decisive for the effectiveness of natural selection: the smaller the population, the greater the effect of chance, and natural selection will function more poorly.

Both Lewontin and Ohta have continued to study genetic variation and have, over a number of decades, made sizable contributions to the continuing development of knowledge in the field. Today, Lewontin and Ohta are Professors Emeriti at Harvard University, MA, USA and the National Institute of Genetics, Mishima, Japan, respectively. During the autumn 2015, AMBIO will publish a Perspective with a more detailed account of the important work of these two pioneers and its relevance for environmental sciences.

Bo Söderström ( $\square)$

Address: The Royal Swedish Academy of Sciences, Box 50005,

10405 Stockholm, Sweden.

e-mail: bo.soderstrom@kva.se 